\title{
XXXI. Electro-optic experiments on various liquids
}

\author{
John Kerr LL.D.
}

To cite this article: John Kerr LL.D. (1882) XXXI. Electro-optic experiments on various liquids, Philosophical Magazine Series 5, 13:81, 248-262, DOI: 10.1080/14786448208627179

To link to this article: http://dx.doi.org/10.1080/14786448208627179

曲 Published online: 28 Apr 2009.

Submit your article to this journal

Џll Article views: 5

Q View related articles ¿ 
to return to its original form; and so we froze water in two $\frac{3}{4}$-inch iron gas-pipes, one round and one which had been flattened when red-hot. The round pipe burst the first time; the flat one did return slightly when thawed, but not enough to prevent its bursting during the second operation.

No doubt most people will not consider this proposal of Mr. Powell's a satisfactory cure for burst pipes ; they would like something which could be fixed in their houses and which would be always safe without further attention. But till such a discovery is made, I think elliptical pipes give the best solution of a problem which has troubled every householder.

As the subject of this paper is of physical as well as general interest, I hope that it may be considered not unworthy of the attention of the Physical Society.

XXXI. Electro-optic Experiments on various Liquids. By JoHN Kerr, LL.D., Free Church Training College, Glasgow.

[Concluded from p. 169.]

Sulphides, $\left(\mathrm{C}_{n} \mathrm{H}_{2 n+1}\right)_{2} \mathrm{~S}$.

13. THESE are purely negative, and rise in power as $n$ 1 increases; they are weaker than the corresponding oxides, but much stronger than the hydroxides.

Ethyl sulphide, not a clean liquid, required the jar and 10 to 20 turns of the plate. The effect was purely negative, strengthened by compression parallel to lines of force, and extinguished by tension.

Butyl sulphide, tried as a nonconductor, gave a very faint effect, which was barely characterizable as negative. Tried then as a conductor, with increasing charges of the jar, it gave a set of brilliant effects, always purely negative.

Amyl sulphide, a feeble photogyre, hardly separating the red and blue at extinction, was rather stronger than the last. It acted well as a nonconductor, giving a clear effect which was purely negative. As a conductor, with increasing charges, it gave increasingly brilliant effects, always negative by both tests.

Ethyl disulphide was also examined, and was found to be purely negative, like the protosulphide, but much stronger. As a nonconductor it gave a pure and moderately strong effect; and as a conductor, with increasing charges of the jar, it gave a fine series of restorations, all purely negative, extinguished perfectly by tension parallel to lines of force. 


\section{Mercaptans, $\mathrm{C}_{n} \mathrm{H}_{2 n+1} \mathrm{HS}$.}

14. These are purely positive, and rise in power as $n$ increases ; they are stronger than the corresponding fatty acids.

Ethyl hydrosulphide acted very well in the plate cell as a conductor, without the jar. The effect from extinction was not very faint, and was slow enough to be charneterized as purely positive.

Butyl hydrosulphide is distinctly stronger. As a nonconductor, it gave a faint but clear effect, which was purely positive; and as a conductor, with increasing charges of the jar, it gave a set of increasingly brilliant effects, which were all strengthened by tension parallel to lines of force, and all weakened to perfect extinction by the proper compression.

Amyl lydrosulphicle, not a clean sample, acted very like the last, but was rather stronger. As a nonconductor (spark $\frac{1}{4}$ inch) it gave a good though faint effect, which was purely positive; and as a conductor, with moderate charges of the jar, it gave an intense restoration, which was always extinguished perfectly by the proper compression parallel to lines of force.

$$
\text { Fomates, } \mathrm{CH}\left(\mathrm{U}_{n} \mathrm{H}_{2 n+1}\right) \mathrm{O}_{2} \text {. }
$$

15. These are active only as conductors, and certainly positive; but the effects are faint and impure, the flame restored in the polariscope boing distorted and streaky. This form of disturbance was noticed already, though in a milder form, in the allyl and ethylene alcohols.

Methyl formate, with moderate charges of the jar, gave an effect which was faint and not at all pure, but certainly positive, strengthened by tension parallel to lines of force, and not sensibly affected by compression.

Ethiyl formate, with good charges of the jar, acted rather better. The effect was strengthened by tension parallcl to lines of force, and much weakened by compression.

Amyl formate acted very like the other two. With moderate charges, the effect was pretty strong and distinctly positive, strengthened by tension, and either weakened or not affected by compression. When the discharges were intense, the disturbance masked the regular effect completely.

$$
\text { Acetates, } \mathrm{C}_{2} \mathrm{H}_{3}\left(\mathrm{C}_{n} \mathrm{H}_{2 n+1}\right) \mathrm{O}_{2} \text {. }
$$

16. These are distinctly positive, and otherwise like the formates; but the effects are purer.

Methyl acetate required the jar. With moderate charges the effect was very abrupt but certainly positive, strengthened 
by tension, and not affected by compression. The Ruhmkorffdischarges gave afterwards an effect which was more purely positive, weakened regularly by compression; but the liquid was now very much disturbed, and there was a large heateffect after a few discharges.

Ethyl acetate acted hardly so well as the last. With moderate charges of the jar, there was a large deformation of the flame, and the regular effect was very faint, not seizable indeed without the hand compensator ; but it was clearly positive, strengthened by tension, and darkened by compression.

Butyl acetate acted much better. As a conductor, without the jar, it gave a clear effect which was purely positive by both tests; and with these feeble discharges the disturbance of the flame was hardly noticed.

Amyl acetate acted still better, but not without the jar. With moderate charges it gave a good effect, which was purely positive, strengthened by tension, and extinguished by compression. With strong discharges the effect was still very fine, and purely positive by both tests, the deformation of the flame being hardly sensible.

$$
\text { Butyrates, } \mathrm{C}_{4} \mathrm{H}_{7}\left(\mathrm{C}_{n} \mathrm{H}_{2 n+1}\right) \mathrm{O}_{2} \text {. }
$$

17. The regular effects in these compounds are purely positive and undisturbed, much stronger and finer than in the acetates.

Methyl butyrate acted well as a conductor, with or without the jar, giving a series of fine effects with increasing charges, and without any apparent disturbance. The effect, weak or strong, was purely positive, extinguished always by the proper compression parallel to lines of force.

Ethyl butyrate, an impure sample, was hardly so strong as the last, requiring the jar and five or more turns of the plate. With good charges the effect was strong, and purely positive by both tests.

Ethyl isobutyrate was much stronger, acting well as a nonconductor, and reminding me of the weaker hydrocarbons, amylene \&c. With strong charges of the jar it gave a strong, slow, and very fine effect, purely positive by both tests, and without sensible disturbance.

Isobutyl isobutyrate gave a fine and purely positive effect as a nonconductor. There was no sensible disturbance even when the electric force was strongest. The action improved evidently for a time as the experiment proceeded, and appeared to be at last as strong as that of benzol. 


$$
\text { Valerates, } \mathrm{C}_{5} \mathrm{H}_{9}\left(\mathrm{C}_{n} \mathrm{H}_{2 n+1}\right) \mathrm{O}_{2} \text {. }
$$

18. These are clearly positive, acting better than the acetates, but not so well as the butyrates.

Methyl valercte, with strong charges of the jar, gave a faint effect which was distinctly positive, strengthened by tension, and weakened by compression. The effect was very impure, the flame being much deformed.

Ethyl valercte was much stronger, and its action was almost perfectly pure. As a nonconductor, it gave a clear, but very faint, restoration from extinction. As a conductor, with increasing charges, it gave a series of fine effects, all purely positive, strengthened by tension, and extinguished by compression.

Amyl valerate, a much stronger photogyre than either of the other two, acted only as a conductor, and required the jar. The regular effect, not strong in any case, was always purely positive by both tests.

$$
\text { Benzoates, } \mathrm{C}_{7} \mathrm{H}_{5}\left(\mathrm{C}_{n} \mathrm{H}_{2 n+1}\right) \mathrm{O}_{2} \text {. }
$$

19. These are purely positive, and abont as strong as the butyrates.

Methyl benzoute acted clearly as a conductor ; but the effect without the jar was too abrupt to be characterized. With small charges of the jar the effect was positive by one test, strengthened by tension, and either weakened or not sensibly affected by compression.

Ethyl benzocte, as a nonconductor, gave a faint but very good restoration, which was purely positive. Tried then as a conductor, with and without the jar, it gave an intense and very slow effect, always purely positive by both tests.

Amyl benzoate, not a clean sample, acted only as a conductor. With moderate charges of the jar, it gave an intense and slow effect which was always purely positive, the extinctionbands being brought in very finely by compression parallel to lines of force.

$$
\text { Nitrates, } \mathrm{C}_{n} \mathrm{H}_{3 n+1} \mathrm{NO}_{3} \text {. }
$$

20. These are weak insulators in comparison with most of the preceding ethers: they are clearly but not very purely positive.

Ethyl nitrate as a conductor, without the jar, gave a restoration which was distinct, but very faint and abrupt. With moderate charges of the jar, the effect was seen to be positive, strengthened by tension, but not affected by compression. With strong charges the effect was still very abrupt, neither pure nor very strong, but certainly positive. 
Butyl nitrate is stronger, giving a sure trace of effect as a nonconductor. Tried as a conductor, with moderate charges of the jar, it gave a strong restoration which was certainly positive, strengthened by tension, but not affected by compression. With very strong charges the effect was brilliant, but still too abrupt, being absolutely unaffected by compression parallel to lines of force; but the contrast between the effects of tension and compression was always perfectly clear.

Amyl nitrate, a feeble photogyre, is very like the last, certainly, but not quite purely, positive. With strongest charges of the jar, the effect was still unmanageably abrupt for the second test; and the liquid was sensibly disturbed.

$$
\text { Nitrites, } \mathrm{C}_{n} \mathrm{H}_{2 n+1} \mathrm{NO}_{2} \text {. }
$$

21. Ethyl nitrite was in solution (10 p. c.), and, when tried with strong charges of the jar, gave a very faint effect which was apparently positive.

Amyl nitrite acted well with moderate charges of the jar. Although the liquid was disturbed sensibly by discharge, the regular effect was seen to be certainly positive, strengthened by tension, and either weakened or not affected by compression.

\section{Other compounds of $\mathrm{C}, \mathrm{H}, \mathrm{O}$.}

22. Aldehyd, $\mathrm{C}_{2} \mathrm{H}_{4} \mathrm{O}$, is negative, and hardly so strong as ethyl alcohol. With Ruhmkorff-discharges, and with precautions against permanent heat-effects, it gave a good restoration, regular though very faint, which was clearly strengthened by compression but not by tension.

Acetone, $\mathrm{C}_{3} \mathrm{H}_{6} \mathrm{O}$, is stronger than the last, clearly positive, but a very feeble insulator. With good charges of the jar it gave a clear effect, not characterizable. With separate discharges of the coil, it gave a fine but still faint restoration from extinction, which was strengthened by tension, but not affected by compression.

Valeral, $\mathrm{C}_{5} \mathrm{H}_{10} \mathrm{O}$, is stronger than the last, and clearly positive. With moderate charges of the jar it gave a good but abrupt effect, strengthened by tension, but not affected by compression. With the coil, the effect was very strong, still not affected by compression; but the contrast between tension and compression was regular and perfectly clear, proving the liquid to be purely positive, but a very weak insulator.

Enanthol, $\mathrm{C}_{7} \mathrm{H}_{14} \mathrm{O}$, is still stronger, and purely positive. As a conductor, without the jar, it gave a clear though faint restoration. With moderate charges of the jar, the effect was good though still faint, strengthened by tension, and extinguished by compression. With strong discharges, the effect 
was more intense but not so pure, the liquid being much disturbed.

Benzoyl hydride, or oil of bitter almonds $\left(\mathrm{C}_{7} \mathrm{H}_{5} \mathrm{O}, \mathrm{H}\right)$, is positive, and a very weak insulator. Without the jar, it gave a clear restoration, too abrupt for the hand-compensator. With moderate charges of the jar, the effect was still extremely quick but certainly positive, strengthened by tension, and not affected by compression.

Ethylene monacetate, $\mathrm{C}_{4} \mathrm{H}_{8} \mathrm{O}_{3}$, ranks with the other acetates and the formates as very impurely positive. With weak discharges of the jar, the effect was too faint to be characterized, or even regularly seized; and with strong discharges of the coil, the liquid was very much disturbed. With moderate charges the effect was to all appearance positive, but very impure at the best.

Methyl salicylate, or oil of wintergreen, $\mathrm{C}_{8} \mathrm{H}_{8} \mathrm{O}_{3}$, is purely positive. As a conductor, without the jar, it gave a sensible but very faint restoration. With strong charges of the jar, the effect was pretty strong and certainly positive, the contrast between tension and compression being clear and regular, though there was no weakening in any ease by compression.

The effect was examined afterwards more closely with the Ruhmkorff-discharges, and with a special eye-piece described in one of my former papers-an acute prism of glass, revolving. regularly round the line of vision, and exbibiting the progress of the phenomenon in time as an extension in space. Viewed in this way, the effect was strengthened by tension as formerly, and was also extinguished regularly and perfectly by compression. I have already referred to this form of experiment as one that would afford a clear proof of the purity of positive or negative character of a dielectric, in cases where the handcompensator gives a sonsible increase of intensity when conspiring with the electric strain, but no sensible decrense when opposing it.

23. Beeswax is purely negative, as are also all the fused fats that have been examined, with the exception of spermaceti, which is strongly positive.

Palm-oil, cacao-lutter, lard, and tallow were tried successively in the fusion-cell as examples of the fats. There was generally a little disturbance by movements in the liquid; but the optical effects vere distinctly and regularly negative, strengthened by compression, and neutralized by tension.

Spermaceti was then tried in the same way, and was found to be purely positive, like sperm-oil among the fixed oils ; but it was much stronger than was expected, stronger indeed than any of the negative fats. As a nonconductor, and with a half 
or quarter turn of the plate, it gave a strong and purely positive effect from extinction, without sensible disturbance of any kind.

A thing noticed in most of the fused fats, and strikingly apparent in the case of spermaceti, was the sluggishness of rise and fall of the electro-optic action. When strong electric force was applied, the optical effect rose gradually to its full intensity, through 1 or nearly 2 seconds; and again, when the apparatus was discharged, the light fell to sensible extinction still more slowly, through 20 or more seconds. This was evidently a thing of the same kind as the gradual rise and fall of effect which I observed long ago in glass; but the electric stress in that case was much more intense, and the duration of the phenomenon much greater, extending even to 20 or 30 minutes.

Beeswar, purified but unbleached, was tried in the same way as the fats, and was found to be purely negative, and almost as strong as spermaceti.

White wax, a colourless and very clear plate in the fusioncell, gave a finer and stronger effect, still purely negative.

\section{Other Compounds of $\mathrm{C}, \mathrm{H}, \mathrm{N}$.}

24. Aniline, $\mathrm{C}_{6} \mathrm{H}_{7} \mathrm{~N}$, is purely negative. Two samples were examined. One of them, tried with plate machine and strongest charges of the jar, gave a faint effect which was purely negative, strengthened by compression, and extinguished by tension. The other sample, which was much deeper in colour, gave no trace of effect with the jar; but with the Ruhmkorff's coil it gave intense restorations, which were purely negative by both tests.

Allylamine, $\mathrm{C}_{3} \mathrm{H}_{5} \mathrm{NH}_{2}$, is very feebly negative. With the Ruhmkorff-discharges, the restorations from extinction were perfectly clear and regular, but very difficult to characterize. Upon the whole, the effect appeared to be certainly negative; but it was very faint, and somewhat impure at the best.

Capronitrile, $\mathrm{C}_{6} \mathrm{H}_{11} \mathrm{~N}$, is feebly but clearly negative. Was tried in the same way as allylamine, and acted rather better, the restorations from extinction being very pure and regular, but still rather difficult to characterize. On the whole, the effect was clearly negative, strengthened by compression, and not affected by tension.

Benzonitrile, $\mathrm{C}_{6} \mathrm{H}_{5} \mathrm{CN}$, is purely and strongly positive. Tried as a conductor, without the jar, it gave an intense and very fine effect, which was strengthened by tension parallel to lines of force, and extinguished perfectly by compression. 
This dielectric reminded me of nitrobenzol; but the action was much less abrupt, and therefore more easily characterized.

Azobenzol, $\mathrm{C}_{12} \mathrm{H}_{0} \mathrm{~N}_{12}$, fuses at $65^{\circ} \mathrm{C}$. into at clean and transparent liquid of a deep red colour. Tried in the fusion-cell as a conductor, with increasing charges of the jar, it gave a series of fine and increasingly brilliant effects, all purely positive.

Diphenylamine, $\mathrm{C}_{12} \mathrm{H} \mathrm{N}_{11}$, fuses at $54^{\circ} \mathrm{C}$. into a transparent, colourless, and very volatile oil. Tried in the fusion-cell as a conductor, with moderate charges of the jar, it gave a pretty strong effect which was purely positive. With strong charges, the regular effect was masked in somo degree by movements in the liquid.

Dimethylaniline, $\mathrm{C}_{8} \mathrm{H}_{11} \mathrm{~N}$, is purely positive. Tried in the plate cell as a conductor, without the jar, it gave a good restoration, which was too quick to be characterized. With small charges of the jar, the effect was very strong and purely positive, strengthened by tension, and extinguished by compression.

Conine gave no trace of effect, even with the strongest discharges of the coil. My sample was almost opaque, and certainly far from pure.

\section{Compounds containing $\mathrm{Cl}$ or $\mathrm{Br}$.}

25. Benzyl chloride, $\mathrm{C}_{7} \mathrm{H}_{7} \mathrm{Cl}$, is clearly positive, but acts only as a conductor. Tried with the jar, it gave no effect with 5 turns, a good effect with 10 or more turns; strengthened always by tension, and sensibly weakened by compression. My sample was not very clean.

Chlorobenzol, $\mathrm{C}_{7} \mathrm{H}_{6} \mathrm{Cl}_{2}$, is purely positive. Acted well, though feebly, as a nonconductor, giving an effect which was strengthened by tension and extinguished by compression.

Ethylene chloride, $\mathrm{C}_{2} \mathrm{H}_{4} \mathrm{Cl}_{2}$, is uncertain. It did give a faint and apparently regular effect with strong discharges; but the disturbance (as in the formic ethers) wits excessive, and made the determination of the sign impossible. 'The results obtained with the next liquid make it probable, if not certain, that the failure in the present case was due to impurity of the sample.

Ethylene bromide, $\mathrm{C}_{2} \mathrm{H}_{4} \mathrm{Br}_{2}$, is purely and strongly positive. Tried as a nonconductor (spark $\frac{1}{8}$ inch), it gave a fine steady restoration, which was strengthened by tension, and extinguished perfectly by compression. As a conductor, with increasing charges of the jar, it gave a series of fine effects, which rose through a large range of intensity, always purely positive. 
Chloroform, $\mathrm{CH} \mathrm{Cl} 3$, is purely negative and pretty strong. As a nonconductor, it gave a fine and purely negative restoration from extinction. As a conductor, with increasing charges, it gave a set of increasingly brilliant effects, always purely negative, extinguished perfectly by tension parallel to lines of force. With strong discharges, there was a fine exhibition of extinction-bands, narrow and strong, brought in by tension parallel to lines of force.

Bromoform, as was expected, is also purely negative, but weaker than chloroform. As a conductor, with the jar, it gave a clear effect with 5 turns, strong with 10 or more turns, and always purely negative.

Acetyl chloride, $\mathrm{C}_{2} \mathrm{H}_{3} \mathrm{OCl}$, is purely negative. As a conductor, with strong charges of the jar, it gave an effect from extinction which was merely strong enough to be characterized as certainly negative, being strengthened by compression, and weakened by tension. With the coil afterwards, there was a certain disturbance produced in the liquid by each discharge ; but the regular effect was clearer and stronger than formerly, and always negative by both tests.

Chloral, $\mathrm{C}_{2} \mathrm{Cl}_{3} \mathrm{OH}$, is positive. As a conductor, with the jar, it gave a good restoration with 10 or more turns of the plate. The effect was regularly strengthened by tension; but not sensibly affected by compression.

Chlorpicrin, $\mathrm{CCl}_{3} \mathrm{NO}_{2}$, is purely negative. Tried as the last, it gave a clear effect with 5 turns, strong with 10 or more turns; and the restored light was always strengthened by compression, and always extinguished by the proper tension.

26. Carbon tetrachloride is purely positive, like the dichloride (formerly examined), but much weaker. Tested for insulation, it gave a good spark ( $\frac{\mathrm{r}}{4}$ inch) from the prime conductor. The optical effect was not proportionably strong, not visible indeed from pure extinction; but with the assistance of the hand-compensator it was brought out very clearly, and was found to be purely positive. There was no advantage obtained by trial of the liquid as a conductor, with or without the jar.

Sulphur chloride is purely positive. As a conductor, with strong changes of the jar, it gave a faint but good effect, which was strengthened by tension, and clearly weakened by compression.

Phosphorus trichloride is purely negative, and stronger than the last. As a conductor, with small charges of the jar, it gave a moderately strong restoration from extinction; and the effect was regularly strengthened by compression, and extinguished by tension. 


\section{Other Compounds and Solutions.}

27. Amyl sulphocyanide, $\mathrm{O}_{6} \mathrm{H}_{11} \mathrm{NS}$, is positive. As a conductor, without the jar, it gave distinct restorations, too faint and quick to be characterized. With strong charges of the jar, the effect was strong, and always distinctly positive though very abrupt, strengthened by tension, and not affected by compression.

Allyl sulphocyanide, or oil of mustard-seed, $\mathrm{C}_{3} \mathrm{H}_{5} \mathrm{CNS}$, is positive. With strong charges of the jar it gave a clear effect, which was too abrupt for the hand-compensator, but apparently positive. With the Ruhmkorff'-discharges the effect was stronger and still very abrupt; but it was certainly positive, strengthened by tension, and not affected by compression. With very strong discharges the liquid was considerably disturbed.

Thialdine, $\mathrm{C}_{6} \mathrm{H}_{13} \mathrm{~N} \mathrm{~S}_{2}$, fuses into a transparent plate at $43^{\circ}$ C. In the fusion-cell, with moderate charges of the jar, it gave a clear effect, pretty strong but very abrupt, certainly negative, being regularly strengthened by compression, and not affected by tension.

Oil of sage is purely positive. The initial extinction was imperfect, the liquid being a weak photogyre. With small charges of the jar, there was a good and purely positive restoration from extinction.

28. Several aqueous solutions were tried in the plate cell, but with no results worth mentioning, except in the two cases of $\mathrm{Cl}$ and $\mathrm{SO}_{2}$.

Chlorine in water is feebly but purely negative. The water was distilled specially for the experiment, saturated with the gas, and then examined immediately. With strong discharges of the coil, there were distinct restorations from cxtinction, very faint but perfectly regular, clearly strengthened by compression, and clearly weakened by tension. The effects were to me barely visible; but they were as distinct as any very faint effects could be; they were also recovered regularly on repetition of the experiment, and with two successive charges of the liquid.

Bromine in water had been examined some time before, and had been entered in my note-book as very apparently, but very feebly, negative.

$\mathrm{SO}_{2}$ in water is also purely negative. The experiment was made immediately after a similar one with water alone; and the contrast between the two was perfect, water being regularly and purely positive, and $\mathrm{SO}_{2}$ in water as regularly and purely negative, but rather feebler. The solution of sulphurous 
acid was not noticeably stronger or weaker electro-optically than the chlorine solution.

\section{Summary.}

29. The princlpal results of the experiments are briefly summed up in the following statements, the numbers of the corresponding articles being attached in each case for reference.

The elementary bodies bromine, phosphorus, sulphur, all in the liquid state, are purely and strongly positive (3).

The hydrocarbons. are nonconductors and purely positive, without known exception. The induction effected up to this time is a mere beginning in such an immense field; but it includes examples of the alcohol radicals or their hydrides, of the benzol series, of the olefines, the paraffins, and the terebenes; also cinnamol and naphthalin. Among the hydrocarbons, great density is generally accompanied by high electro-optic power (4).

The common alcohols are negative as a class. From the higher members downwards, the negative electro-optic power diminishes regularly, till it passes, in the last step of the series (ethyl to methyl), from feeble negative to feeble positive. Distilled water also is distinctly positive (5).

Of the series of fatty acids, those liquid at ordinary temperatures are in constant opposition of sign to the corresponding alcohols ; they are also very much stronger. Of those that are solid at ordinary temperatures, two have been examined in a state of fusion, and are found, on the contrary, to be strongly negative $(6,7)$.

Of other alcohols and acids, the allyl, benzyl, cinnyl alcohols are negative; the diatomic glycol and the triatomic glycerin are feebly and impurely negative; phenol is distinctly positive, though in its chemical relations rather an alcohol than an acid; the oleic and lactic acids are both positive, the former strong, the latter very feeble (8).

The oxides of ethyl and amyl are purely negative, and stronger than the corresponding alcohols. Amyl oxide is a moderately good insulator, and one of the best negative dielectrics yet known (9).

The haloid ethers are purely positive, and rise in power from one series to another in the order I, Br, Cl ; they generally rise also in power from lower members upwards. Some of them show an extraordinary increase of insulating and electro-optic powers as the experiment proceeds. Amyl chloride is one of the best positive dielectrics known; amyl bromide also is very strong $(10,11,12)$. 
The sulphides of the alcohol radicals are purely negative and weaker than the corresponding oxides, but much stronger than the alcohols. From lower members upwards they rise in electro-optic power (13).

The hydrosulphides are purely positive, and stronger than the corresponding fatty acids. From lower members upwards they rise in power (14).

Of the compound ethers, all that bave been examined are clearly positive; and they include examples of the formates, acetates, butyrates, valerates, benzoates, nitrates, and nitrites. The optical effects are pure in the butyrates, but more or less disturbed in the others, impure in the acetates, very impure in the formates $(15-21)$.

The following table contains a few additional bodies which are found to be electro-optically active. The optical effects are neither impure nor very weak, except in the cases that are so marked. 'The positive and the negative dielectries appear' always in the left-hand and right-hand columns respectively.

Compounds of $\mathrm{C}, \mathrm{H}, \mathrm{O}(22,23)$.

Acetone (wk).

Valeral.

Enanthol.

Benzoyl hydride.

Methyl salicylate

Ethene monacetate (imp.)

Spermaceti.

Benzonitrile.

Compounds of $\mathrm{C}, \mathrm{H}, \mathrm{N}(24)$.

Azobenzol.

Diphenylamine.

Dimethylaniline.
Aldehyde (wk).

Palm-oil.

Cacao butter.

Lard.

Tallow.

Beeswax.

Compounds containing $\mathrm{Cl}$ or $\mathrm{Br}(25,26)$.

Chlorobenzol.

Benzyl chloride.

Ethylene dibromide.

Chloral.

Carbon tetrachloride.

Sulphur chloride.
Aniline.

Capronitrile (wk).

Allylamine (wk).

\section{Other Compounds and Solutions $(27,28)$.}

Amyl sulphocyanide. Allyl sulphocyanide (wk). Oil of sage.
Chloroform.

Bromoform.

Chlorpicrin.

Acetyl chloride (wk).

Phosphorus trichloride.

Thialdine.

$\mathrm{Cl}$ in water (wk).

$\mathrm{SO}_{2}$ in water (wk). 
Concluding Remarks.

30. Bodies under electric stress are lirefringent, with reference to line of electric force as axis. - This property has now been exemplified in such a number and such a variety of dielectrics, that it ought to be admitted as a general property of matter. There do occur cases (in the formic ethers \&c.) in which the purity of electro-optic double refraction is lost : but these are no true exceptions to the general statement; they are merely cases of disturbance, cases in which the regular effect is masked, wholly or partly, by the presence of other effects. Gross movements and heat appear to be the principal causes of disturbance.

Dielectrics are divisible into two classes, the positive and the negative, corresponding to the two so-named classes of uniaxal crystals. -This also has been abundantly confirmed by experiment, not as a rough adaptation of the notions and terms of Optics to our present subject, but as an exact physical truth. Except in cases of impure action or of mixed optical effects, cases which are comparatively rare in fact, the experimental contrast between the two kinds of dielectric is always as pure to sense, and under any tests yet applied, as is the contrast between quartz and Iceland spar. The two finest examples of the positive class are carbon disulphide and amyl chloride ; and these are far superior in power to any negative dielectric yet discovered. As interesting examples of the negative class, I select the following four, arranging them (according to my samples) in the order of descending powers :-amyl oxide, chloroform, capryl alcohol, aniline.

Among bodies yet examined, the positive dielectrics are more numerous than the negative. - Of 13 amyl compounds, 10 are purely positive, and 3 purely negative; and similarly in other cases. Br, P, S, the only elements yet examined, are all purely positive.

The electro-optic and chemical characters of compounds are closely connected.--The hydrocarbons are positive, the fixed oils and fats negative, the fatty acids positive, the alcohols negative, \&c.

The electro-optic character of a chemical compound is not determined by its empirical formula.-Isomeric bodies are sometimes electro-optically similar, and sometimes not. This is shown in the four following sets of isomers or polymers: propionic acid, methyl acetate, ethyl formate-all positive ; butyl alcohol and ethyl oxide, both negative; aldehyde, butyric acid, ethyl acetate-the first negative and the other two positive; allyl alcohol, acetone, caproic acid, methyl valerate-the first negative and the others positive. 
Every chemically homologous series of bodies yet examined exhibits a certain constancy of electro-optic character.-Thus, the oxides, hydroxides, and sulphides of the alcohol radicals are negative ; while the hydrosulphides, the iodides, bromides, chlorides, butyrates, benzoates, \&c. are positive.

Through each of these homologous series, there is generally a progression of electro-optic power, the higher members being the stronger.-The common alcohols and the fatty acids (liquid at ordinary temperatures) afford very good illustrations. The two series of bodies are negative and positive respectively; and in each of the series, from member to higher member, there is a regular increase of absolute electro-optic power. The progression is not maintained in the higher parts of the acid series, the palmitic and stearic acids being (as the other fats are) strongly negative.

This progression, in singular cases, takes the form even of a change of sign.-The only examples yet known are afforded by the two last mentioned series, which begin thus :

$$
\left.\begin{array}{l}
\text { methyl alcohol }+ \\
\text { ethyl alcohol }-
\end{array}\right\} \quad\left\{\begin{array}{l}
- \text { formic acid } \\
+ \text { acetic acid }
\end{array}\right.
$$

This occurrence of contrary changes of sign at corresponding points of the two series is surely a very remarkable fact. The two following inferences are perhaps worthy of notice.

Oxygen is probably a negative dielectric.-Putting $n=0$ in the general formulas of alcohol and acid, we obtain $\mathrm{H}_{2} \mathrm{O}$ and $\mathrm{O}_{2}$ as the starting-points of the two series. Water and oxygen should therefore have the same signs as methyl alcohol and formic acid respectively. As far as water is concerned, the inference is confirmed already by experiment.

Our terms "positive" and "negative," borrowed originally from Physical Optics, appear now to be terms intrinsically appropriate to the subject.-For in the acids we see a regular increase of positive power showing itself at one point of the series as a passage from - to $t$, and, again, in the alcohols a regular increase of negative. power showing itself at one point as a passage from + to - .

31. Electro-optic double refraction is brought about by a special state of the dielectric, a state of essentially divectional strain, which is a concomitant and a condition of the maintenance of electric stress. This appears to me to be a principle that must underlie any admissible explanation of the phenomena. The carefully grounded and very valuable conceptions of Faraday and Maxwell point in this one direction; and the simple facts of the case are, of themselves, quite decisive. What clearer manifestations of directional strain could be Plil. Mag. S. 5. Vol. 13. No. 81. April 1882. Y 
desired than those that are presented here? There is, first, the directional transmission of electrio force from limit to limit of the field; there is, secondly, the special dioptric action of the force-transmitting medium, an action precisely similar to that of glass directionally strained by tensions or pressures; and there is, finally, the directional rupture of the dielectric when the intensity of the transmitted force reaches a critical value characteristic of the medium.

I insist upon the essentially directional character of electric strain, in opposition to a theory of electro-optic action which has been advanced by Professor Quincke*.

Electro-optic double refraction may be attributed to a quasicrystalline and uniaxal structure, which is maintained by electric stress in the medium. I advanced this view of the phenomena in my first paper on the subject; and I still believe that it contains a germ of the truth. I think indeed that, to any one engaged with the facts of Electro-Optics, there is no theoretical conception that can present itself more naturally than that of an electrically induced structure, a regular arrangment of polarized molecules, roughly illustrated by the common " magnetic curves."

XXXII. On the Electric Resistance of Carbon under Pressure. By Professor Silvanus P. Thompson, B.A., D.Sc. $\dagger$

\$1. T T has often been stated that the electric resistance of carbon decreases when subjected (1) to an increase of temperature, (2) to a mechanical compression. The first of these statements has been verified by so many authorities that there can hardly be any question of its correctness. The second I believe to be wholly misleading; for some careful experiments that I have lately made lead to the conclusion that the effect of mechanical compression upon the electric resistance of dense carbon is almost, if not quite, nil, and that what has been mistaken for an increase in electric conductivity is in reality merely better contact at the points of junction with the circuit.

$\S 2$. A preliminary experiment to test whether the alleged decrease of resistance by pressure was due to a true increase in specific conductivity or to better end-contact, was made in the following manner:- $\Lambda$ thin cylindrical rod of Carre's dense artificial carbon (such as is used in electric lamps) was taken, its length being 72 centimetres. At a point about one

* Phil. Mrag. for July 1880, p. 37.

+ Communicated by the Physical Society, having been read at the Meeting on February 25, 1882. 